\title{
Impacts on thin elastic sheets
}

\author{
By Romain Vermorel, Nicolas Vandenberghe and Emmanuel \\ VILLERMAUX \\ IRPHE, CNRS - Aix-Marseille Université, 49, rue Frédéric Joliot-Curie \\ 13384 Marseille Cedex 13, France
}

We study transverse impacts of rigid objects on a free elastic membrane, using thin circular sheets of natural rubber as experimental models. After impact, two distinct axisymmetric waves propagate in and on the sheet. First a tensile wave travels at sound speed leaving behind the wave front a stretched domain. Then, a transverse wave propagates on the stretched area at a lower speed. In the stretched area, geometrical confinement induces compressive circumferential stresses leading to a buckling instability, giving rise to radial wrinkles. We report on a set of experiments and theoretical remarks on the conditions of occurrence of these wrinkles, their dynamics and wavelength.

Keywords: membrane, impact, buckling, wrinkling

\section{Introduction}

When a thin elastic sheet is impacted transversely by a rigid body, it deforms locally out of its plane. Depending on the nature of the material and the strength of the impact, the sheet may be perforated, it may deform permanently, or it may present radial or circumferential cracks (Backman \& Goldsmith 1978). An understanding of these phenomena requires a knowledge of the global response of a sheet to localized impacts.

Sheets with a small thickness have a weak intrinsic bending rigidity. Very thin sheets such as clothes or biological membranes can thus freely bend and they often exhibit wrinkles (Cerda et al. 2004). If the sheet is stretched, this is no longer the case since any transverse motion is accompanied by a restoring force proportional to the tension. On the other hand, if the sheet is compressed, a buckling instability develops and the membrane wrinkles.

We consider the problem of a free standing axisymmetric membrane impacted transversely at its center. A static version of this problem, namely a membrane clamped at its boundaries deformed by a punctual force, has been considered by Begley \& Mackin (2004). In this static configuration the membrane remains axisymmetric for any force, with no wrinkling. When a free standing membrane (i.e. a membrane with no initial stress) is impacted, tensile stresses develop as a result of the impact. This problem is more complicated than the classical drum problem (Graff 1975) in which the variation of in plane stresses due to the impact itself are neglected. Phoenix \& Porwal (2003) have reviewed previous results on the impacts on free standing membranes in the context of fibrous system (e.g. textile etc...). They also developed a model for the ballistic response of an axisymmetric free standing membrane neglecting compressive circumferential stresses. However, as seen on figures 2 and 3, when a free standing membrane is impacted, it buckles 
(i)

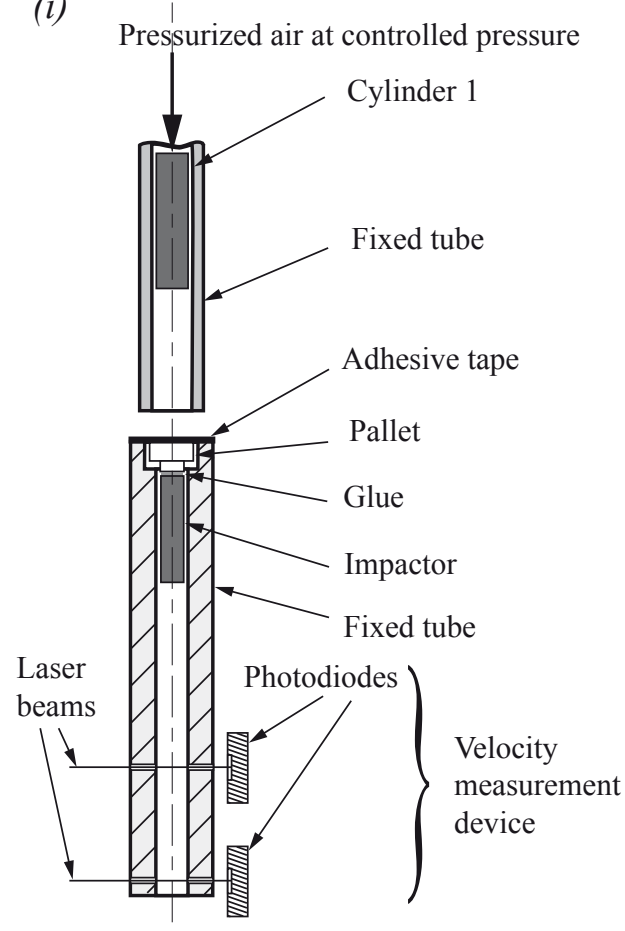

(ii)

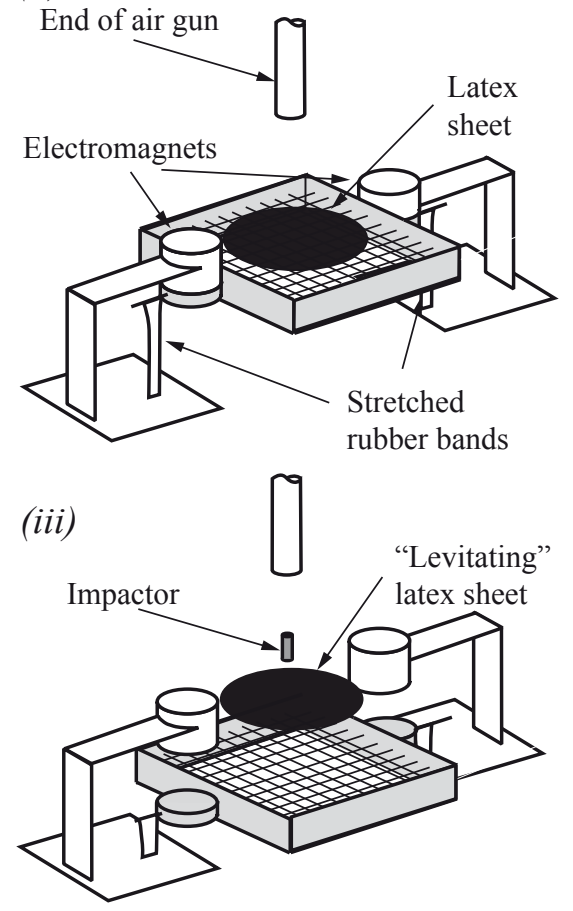

Figure 1. Experimental setup.(i)A two stage gas gun is used to launch impactors. When the cylinder 1 hits the pallet, the adhesive tape is teared off, the pallet is accelerated and the slightly glued impactor is released. (ii) The impacted sheet stands on a netting stretched on a frame. (iii) When the gas gun is triggered, the electromagnets are switched off and the frame is pulled by two rubber bands. The impactor hits a free sheet.

and radial wrinkles appear. The aim of the present work is to describe the sequence of events inducing this instability. First we describe the axisymmetric stress field resulting from the impact and we show that a region of the membrane experiences circumferential compression. We then study the development of the buckling instability leading to the formation of radial wrinkles, and we propose a model predicting their wavelength.

\section{Experimental set-up}

We use a vertical gas gun to launch steel cylinders and spheres of radius $r_{i}=$ $2.25 \mathrm{~mm}$ (figure 1). Unless otherwise noted steel cylinders of length $27 \mathrm{~mm}$ and mass $3.3 \times 10^{-3} \mathrm{~kg}$ are used for the measurements. A cylinder is accelerated by pressurized air inside a $40 \mathrm{~cm}$-long tube. Beneath this tube stands a pallet to which we slightly glue the impactor (cylinder or sphere). As the cylinder hits the pallet the impactor is released. The impactor is guided in a second tube of length $10 \mathrm{~cm}$. At the end of this tube two laser beams are used to measure the speed of the impactor. This two stage design ensures that the latex sheet is not perturbed by 
the gas ejected from the gun. The speed of the impactor can be adjusted with a pressure regulator.

The latex sheet stands horizontally on a netting stretched on an open frame. The open frame is maintained approximately $10 \mathrm{~cm}$ below the end of the second tube by two electromagnets which are synchronized with the gas gun. When the electromagnets are switched off, two rubber bands violently pull the frame down and free the latex sheet. The latex sheet falls down under the action of gravity but the characteristic time scale of its fall is much longer than any other time scale in the problem. Thus the impactor hits a perfectly free "levitating" latex sheet. As discussed in section $6 \mathrm{c}$ the boundary conditions at the outer radius of the membrane do not play a crucial role in the study, and the main purpose of our setup is to ensure that the latex sheet is stress-free when it is hit by the impactor.

The sheets are cut from natural latex rubber sheets of different thicknesses from $0.10 \mathrm{~mm}$ up to $0.30 \mathrm{~mm}$. The radius of the sheets is typically $r_{0}=60 \mathrm{~mm}$. Static stretching tests reveal that in the range of stretching between 0 and 100 percent, the elastic behavior of the rubber remains linear (within 3 percent) with a Young modulus $E=1.5 \mathrm{MPa}$ and no significant hysteretic behaviour i.e. stress softening of the rubber (Bouasse and Carrière 1903 and Mullins 1947) are observed. In all our experiments the strain does not exceed $60 \%$. The density of the rubber is $\rho=990 \mathrm{~kg} \mathrm{~m}^{-3}$, the Poisson modulus is $\nu=0.5$ and thus the nominal wave speed for in plane disturbances is $c=\sqrt{E / \rho\left(1-\nu^{2}\right)}=45 \mathrm{~m} \mathrm{~s}^{-1}$.

Our diagnostics are based on quantitative image analysis, resolved in time. The typical time scale is $r_{0} / c \approx 1.3 \mathrm{~ms}$. We use a Photron high-speed video camera to record movies at typical frame rates of 7,000 up to 90,000 frames per second. For side views, we use direct lighting with a black background. For movies showing a view from bottom a mirror is placed underneath the supporting device and the sheet is illuminated from above. When needed, regularly spaced marks are drawn on the sheet to follow the motion of the material points.

As a complement, to document the impact phenomenology, we also conduct experiments using narrow latex strips instead of the circular latex sheets. The thickness of the strips is $0.10 \mathrm{~mm}$, their length is $100 \mathrm{~mm}$ and their width is $4 \mathrm{~mm}$.

\section{Phenomenology}

When a rigid body impacts the center of the thin elastic disc at velocity $V$ (figure $2 a$ ), a tensile front sets out from the impact point and propagates radially toward the edge of the sheet at speed of sound $c$. In the stretched area, material points move toward the center of the sheet. As the impactor pulls the membrane, with no observed sliding of the rubber against the impactor, a cone expands toward the edge of the sheet at velocity $\bar{U}$ (with $\bar{U}<c$ ) in the laboratory frame. In the stretched area, tensile stress in the radial direction is accompanied by contraction in the direction normal to it. Thus compressive circumferential stresses develop in the stretched area and the sheet buckles, giving rise to wrinkles (figure 3).

Experiments show that the buckling instability selects a wavelength rather than a number of folds. Indeed, as the transverse wave front propagates the perimeter and width of the compressed area increase with time and so does the number of folds, which results in a conservation of the buckled wavelength. As can be seen 

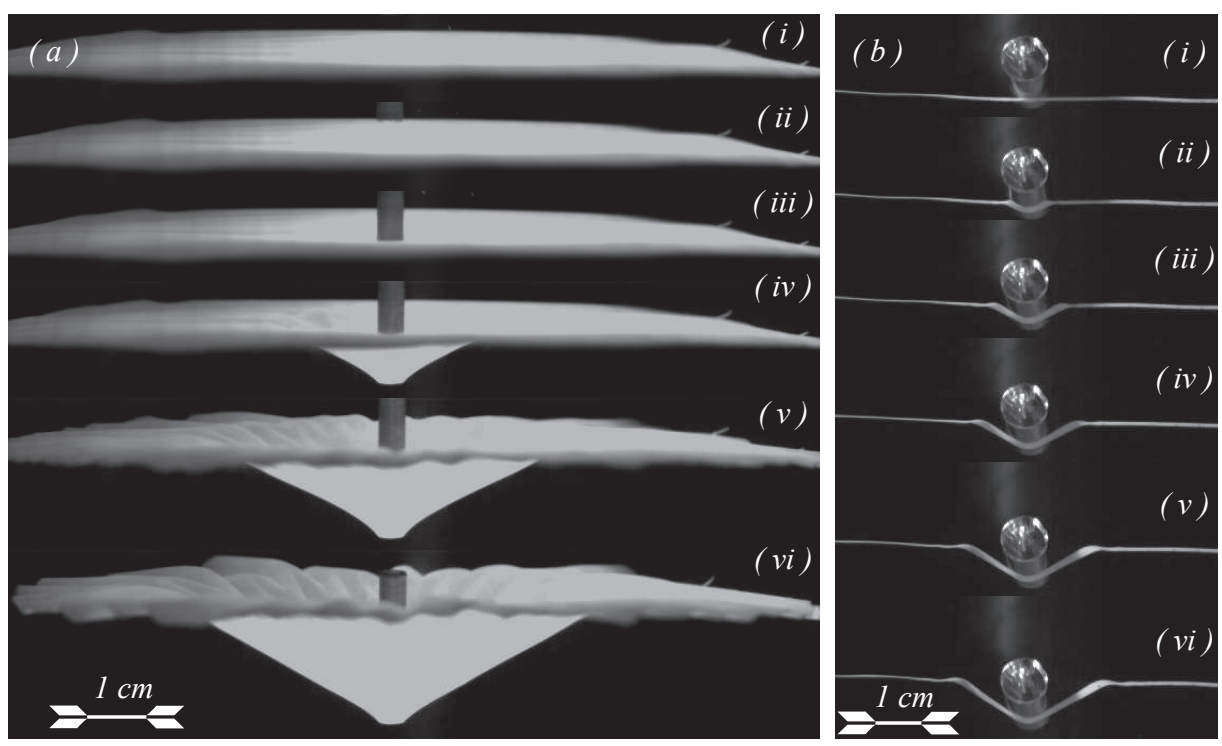

Figure 2. (a): Impact of a steel cylinder on a thin rubber sheet of radius $r_{0}=60 \mathrm{~mm}$ and thickness $h=0.10 \mathrm{~mm}$. The timestep between two frames is $1.33 \mathrm{~ms}$. (i) to (iii) The cylinder of radius $r_{i}=2.25 \mathrm{~mm}$ impacts the free "levitating" sheet at velocity $V=5.8 \mathrm{~m} \mathrm{~s}^{-1}$. $(i v)$ After impact a cone expands. ( $v)$ to $(v i)$ The propagation of both tensile and transverse waves yield an in plane stress field that will eventually induce a buckling instability. A movie showing the dynamics is included in the supplementary materials. (b): Impact of a free falling rod on a thin rubber strip of thickness $0.10 \mathrm{~mm}$, length $100 \mathrm{~mm}$ and width $4 \mathrm{~mm}$. The timestep between two frames is $0.50 \mathrm{~ms}$. (i) The rod impacts the free "levitating" strip with velocity $V=3.7 \mathrm{~m} \mathrm{~s}^{-1}$. (ii) to $(v i)$ After impact a V-shaped area expands.

on figure 4 new folds are generated during the propagation of the transverse wave front, so that the wavelength does not change with time.

We also study the one dimensional version of the problem, the impact on a thin elastic strip (figure $2 b$ ). The phenomenology is similar except, de facto, for the formation of wrinkles: first a tensile front propagates away from the impact point and then, as the impacting body drags the strip out of its plane, a transverse "V-shape" expands in the stretched material.

\section{Wave propagation after impact}

\section{(a) Wavefronts resulting from an impact on a $1 D$ elastic string}

An elastic string has a density $\rho$ and Young modulus $E$. The string length $\ell$ is such that $\ell \gg c t$, in which $c=\sqrt{E / \rho}$ is the nominal wave speed for longitudinal disturbances (Love 1944) and $t$ the typical time of observation after impact. The free string is impacted transversely at the point $x=0$. The mass of the impacting body is very large compared to the mass of the string, and the impacting velocity $V$ is such that $V / c \ll 1$. In that limit, the projectile is not decelerated.

When the string is impacted, a longitudinal wave propagates at speed $c$. Behind this wave the stretching $\epsilon$ (to be determined) is taken as constant and uniform. 


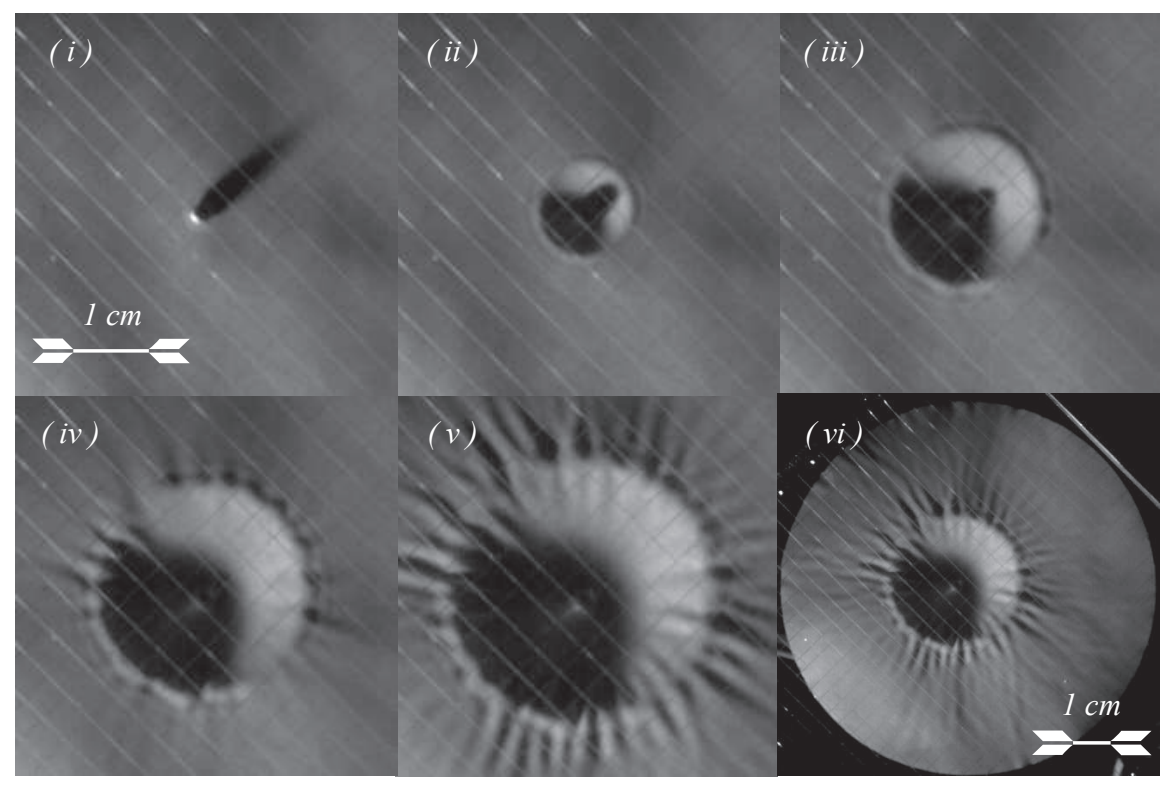

Figure 3. Bottom view of the impact of a steel ball of radius $2.25 \mathrm{~mm}$ on a thin rubber sheet of radius $55 \mathrm{~mm}$ and thickness $0.10 \mathrm{~mm}$. The timestep between two frames is $0.50 \mathrm{~ms}$. (i) to $(i i)$ : As the ball impacts the sheet at speed $V=5.2 \mathrm{~m} \mathrm{~s}^{-1}$, the transverse wave front propagates radially. (iii) to $(v)$ : material points are pulled towards the center of the sheet and a circumferential compressive stress triggers a buckling instability selecting a well defined wavelength. $(v i)$ : zoomed out view of $(v)$. A movie showing the dynamics is included in the supplementary materials.

Material points are thus moving towards the impact point at speed $W=-c \epsilon$. Behind the tensile front, material points are moved transversely and a "V-shaped" area expands (figure $2 b$ ). The base of this area travels at the speed of propagation of transverse disturbances. We assume that the stretching is $\epsilon$ also in the $\mathrm{V}$-shaped area. In the frame of material points, the equation for the transverse displacement $\xi(x, t)$ is

$$
\rho A \frac{\partial^{2} \xi}{\partial t^{2}}=\frac{\partial(T \sin \phi)}{\partial x}
$$

where $A$ is the cross section area and $T=E A \epsilon$ is the tension in the string. $\phi(x, t)$ is the angle between the local tangent and the $x$-axis and $(1+\epsilon) \sin \phi=\partial \xi / \partial x$. Thus the speed of transverse disturbances in the frame of material points is

$$
U=c \sqrt{\frac{\epsilon}{1+\epsilon}}
$$

At time $t$, the transverse wavefront reaches the material point $x_{F}=U t$. This point has travelled a distance $W\left[t-t_{l}\left(x_{F}\right)\right]$ where $t_{l}\left(x_{F}\right)=x_{F} / c$ and thus its position in the laboratory frame is

$$
\bar{x}_{F}=x_{F}-c \epsilon\left[t-t_{l}\left(x_{F}\right)\right]=x_{F}(1+\epsilon)-c \epsilon t
$$




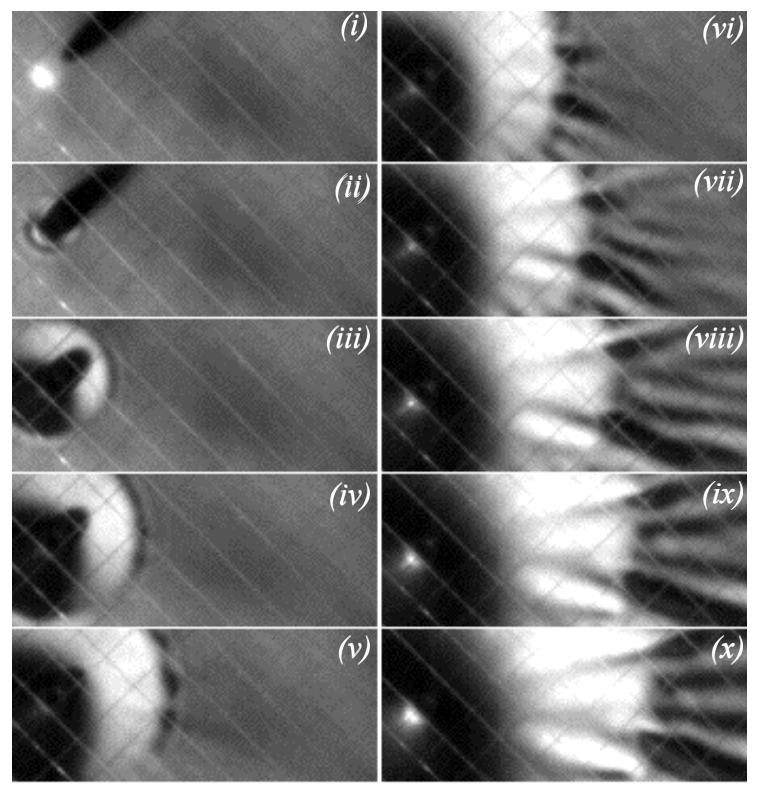

Figure 4. Close bottom view of the transverse wavefront after impact of a steel ball at $V=5.2 \mathrm{~m} \mathrm{~s}^{-1}$ on a thin rubber sheet. Time goes by steps of $0.70 \mathrm{~ms}$. Wrinkles appear at different stages so that the wavelength of the wrinkling pattern is constant.

To relate the impactor speed with other quantities, we use Pythagoras' theorem

$$
\bar{x}_{F}^{2}+(V t)^{2}=(1+\epsilon)^{2} x_{F}^{2}
$$

Substituting $\bar{x}_{F}, x_{F}$ and $U$, we obtain an equation for $\epsilon$

$$
[\sqrt{\epsilon(1+\epsilon)}-\epsilon]^{2}(c t)^{2}+(V t)^{2}=\epsilon(1+\epsilon)(c t)^{2}
$$

Interestingly, the leading contribution to the motion of the transverse wave front is the motion of the material points towards the impact point at speed $-c \epsilon$. We obtain $\epsilon$ and the speed of the transverse wave front in the laboratory frame $\bar{U}=\bar{x}_{F} / t$

$$
\begin{aligned}
\epsilon & =\left(\frac{V}{\sqrt{2} c}\right)^{4 / 3}+\left(\frac{V}{\sqrt{6} c}\right)^{2}+\mathcal{O}\left((V / c)^{2}\right) \\
\frac{\bar{U}}{c} & =\left(\frac{V}{\sqrt{2} c}\right)^{2 / 3}-\frac{5}{6}\left(\frac{V}{\sqrt{2} c}\right)^{4 / 3}+\mathcal{O}\left((V / c)^{2}\right)
\end{aligned}
$$

Figure 5 shows the measured dependence of the transverse wave front velocity on the impacting velocity. Equation 4.7 agrees well the measurements, but the theory overestimates the transverse front velocity. This may come from the 2D geometry of the latex rubber strips while we used a 1D model for strings. Indeed a careful examination of the images shows that the base of the cone connects smoothly rather than angularly with the straight section. This connection occurs over a a length of a few millimeters (the width of the strip is $4 \mathrm{~mm}$ ). Nevertheless, results clearly show that the mechanism we propose for the wavefront propagation after impact is the good scenario. 

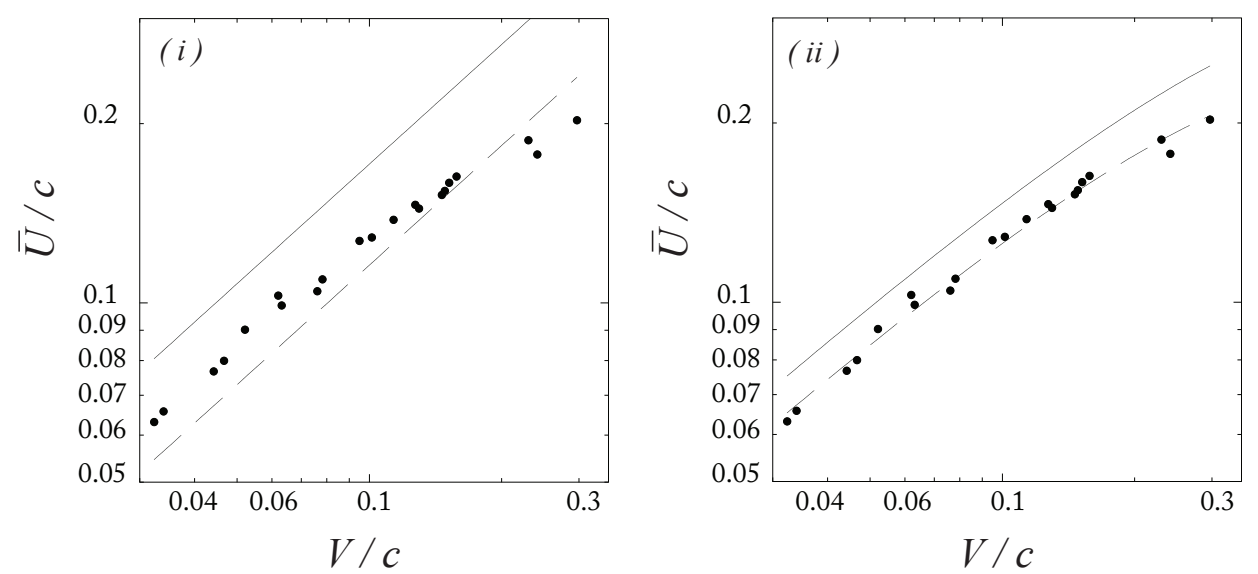

Figure 5. Transverse wave front velocity in the laboratory frame vs impacting velocity, measured for a thin strip. The solid lines stand for the theoretical predictions with no adjustable parameter while the dotted lines stand for the fit with an adjusted prefactor. (i) First order in $\epsilon$ prediction. (ii) Second order in $\epsilon$ prediction.

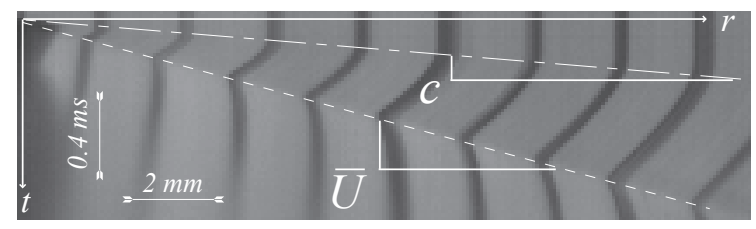

Figure 6. Space-time reconstruction of the motion of the material points on an impacted membrane taken from a high speed movie. The longitudinal wavefront propagates at the speed of sound $c$. The transverse wave front propagates at a constant speed $\bar{U}$ with $\bar{U}<c$.

\section{(b) Waves resulting from an impact on a $2 D$ elastic membrane}

The problem of the impact on a circular membrane is more complicated because circular waveforms do not maintain their shape as they propagate. The propagation mechanism is however the same as for the strings discussed in section 4a. First a tensile front propagates at the speed of sound in the material. Then a cone grows in a stretched area and thus it must obey the equation of propagation for transverse perturbations. Our objective here is to provide an approximate form for the stress field in the area of the tensile wave. This stress field will later be used to study the development of the wrinkling instability.

A circular elastic membrane has density $\rho$, Young modulus $E$ and Poisson ratio $\nu$. The radius of the membrane is such that $r_{0} \gg c t$, in which $c$ given by $c^{2}=$ $E /\left[\left(1-\nu^{2}\right) \rho\right]$ is the nominal wave speed for in plane disturbances and $t$ is the typical time of observation after impact. The initially stress and strain free membrane is transversely impacted at the point $r=0$. We consider that the mass of the impactor is much larger than the mass of the membrane, a limit for which the projectile deceleration can be neglected. The impacting velocity $V$ is such that $V / c \ll 1$. 

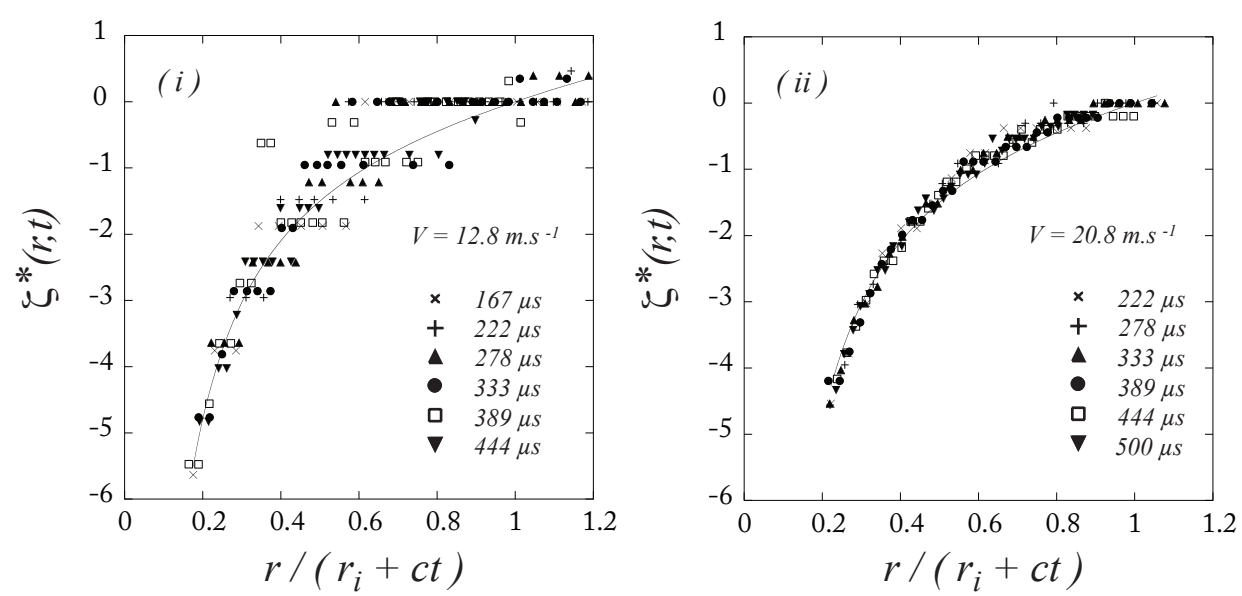

Figure 7. Radial displacement vs radial coordinate. We note $\zeta^{*}(r, t)=\zeta(r, t) /\left[\alpha\left(r_{i}+c t\right)\right]$. The solid line stand for the form given by (4.9) where the parameter $\alpha$ has been fitted. (i) Measurements performed for the impacting velocity $V=12.8 \mathrm{~m} \mathrm{~s}^{-1}$ at different times after impact. (ii) Measurements performed for the impacting velocity $V=20.8 \mathrm{~m} \mathrm{~s}^{-1}$.

The radial displacement $\zeta(r, t)$ is given by the wave equation

$$
\frac{1}{c^{2}} \frac{\partial^{2} \zeta}{\partial t^{2}}=\frac{1}{r} \frac{\partial}{\partial r}\left(r \frac{\partial \zeta}{\partial r}\right)-\frac{\zeta}{r^{2}}
$$

This equation has no simple solution and we use a quasistatic approximation

$$
\zeta(r, t)=\alpha\left(r_{i}+c t\right)\left(\frac{r}{r_{i}+c t}-\frac{r_{i}+c t}{r}\right)
$$

where $\alpha$ is a dimensionless constant to be determined. The form for the displacement corresponds to the solution of the static problem with the boundary condition $\zeta=0$ at the tensile wave front in $r=r_{i}+c t$. This solution cancels the right hand side of equation (4.8). The radial strain is

$$
\epsilon_{r}(r, t)=\frac{\partial \zeta}{\partial r}=\alpha\left(1+\left(\frac{r_{i}+c t}{r}\right)^{2}\right)
$$

and thus at the tensile wave front $\epsilon_{r}=2 \alpha$. We measure the radial displacement by recording the motion of regularly spaced marks drawn on the latex sheet. Figure 7 shows that the form of equation (4.9) fits the measurements.

The base of the cone propagates in a stretched area and we find experimentally that the propagation speed does not vary in time. Transverse displacements $\xi(r, t)$ for a membrane are ruled by

$$
\rho \frac{\partial^{2} \xi}{\partial t^{2}}=\frac{1}{r} \frac{\partial}{\partial r}\left(r \sigma_{r} \sin \phi\right)
$$

where $\phi$ is the angle between a meridian line and the local tangent to the membrane in the radial direction and we have $\sin \phi \approx \partial \xi / \partial r$. The stresses are

$$
\sigma_{r}=\frac{E}{1-\nu^{2}}\left(\epsilon_{r}+\nu \epsilon_{\theta}\right) \text { and } \sigma_{\theta}=\frac{E}{1-\nu^{2}}\left(\epsilon_{\theta}+\nu \epsilon_{r}\right)
$$


where $\epsilon_{r}=\partial \zeta / \partial r$ is the radial strain and $\epsilon_{\theta}=\zeta / r$ the circumferential strain. The radial strain is positive while the circumferential strain is negative.

To relate the strain in the stretched domain with the speed of the impactor we use the same geometrical argument as for the impacted string. The radial strain in the cone is taken as uniform and equal to $\epsilon_{c}=\epsilon_{r}\left(r_{c}, t\right)$. The geometrical relation is

$$
\left(1+\epsilon_{c}\right)^{2} r_{c}^{2}(t)=(V t)^{2}+(\bar{U} t)^{2}
$$

where $r_{c}(t)=r_{i}+U t$ is the (Lagrangian) coordinates of the transverse wave front and $\bar{U} t=r_{c}(t)+\zeta\left(r_{c}, t\right)$ is the position of the front in the laboratory frame. The right hand side in equation (4.13) is based on the assumption that the membrane shape is conical, a good approximation as seen from figure $2 a$.

We assume that the transverse wave front is far from the impactor, i.e. $U t \gg r_{i}$, we have

$$
\zeta\left(r_{c}, t\right)=\alpha\left(r_{i}+c t\right)\left(\frac{r_{i}+U t}{r_{i}+c t}-\frac{r_{i}+c t}{r_{i}+U t}\right) \approx \alpha c t\left(\frac{U}{c}-\frac{c}{U}\right)
$$

In this expression $\alpha$ and $U$ are unknown. At the base of the cone, for large $t$, the radial strain deduced from the approximation for $\zeta$ is

$$
\epsilon_{c}=\alpha\left[1+\left(\frac{r_{i}+c t}{r_{i}+U t}\right)^{2}\right] \approx \alpha\left[1+\left(\frac{c}{U}\right)^{2}\right]
$$

When $\sigma_{r}$ is constant in equation (4.11), transverse displacement waves propagate at speed $c_{t}=c \sqrt{\epsilon_{r}+\nu \epsilon_{\theta}}$ (see e.g. Graff, 1975). Thus we have

$$
U=c \sqrt{\alpha\left(1+\nu+(1-\nu)\left(\frac{c}{U}\right)^{2}\right)}
$$

which gives

$$
\alpha=\frac{\left(\frac{U}{c}\right)^{4}}{1-\nu+(1+\nu)\left(\frac{U}{c}\right)^{2}}
$$

In equation (4.13), we have at leading order

$$
\left(1+\frac{2}{1-\nu}\left(\frac{U}{c}\right)^{2}\right)(U t)^{2}=(U t)^{2}-\frac{2}{1-\nu}\left(\frac{U}{c}\right)^{2}(U t)^{2}+(V t)^{2}
$$

and thus

$$
\frac{U}{c}=\left(\frac{1-\nu}{4}\right)^{1 / 4}\left(\frac{V}{c}\right)^{1 / 2}
$$

Figure 8 shows experimental measurements of the transverse wave front versus impactor speed. The model, which assumes $V / c \ll 1$, overestimates the propagation speed but its dependence on the impacting speed is in good agreement. The model also assumes that $U t \gg r_{i}$, but experiments show that the final speed of the transverse wave front is attained for $r$ very close to $r_{i}$ (see figure 6 ). The measurements conducted for different sheet thicknesses $h$ show that the transverse front velocity does not depend on $h$ for $0.10<h<0.30 \mathrm{~mm}$, as expected. For larger thicknesses, experiments reveal that the bending rigidity of the latex rubber sheet cannot be neglected, and discrepancies with the elastic membrane model are observed. 

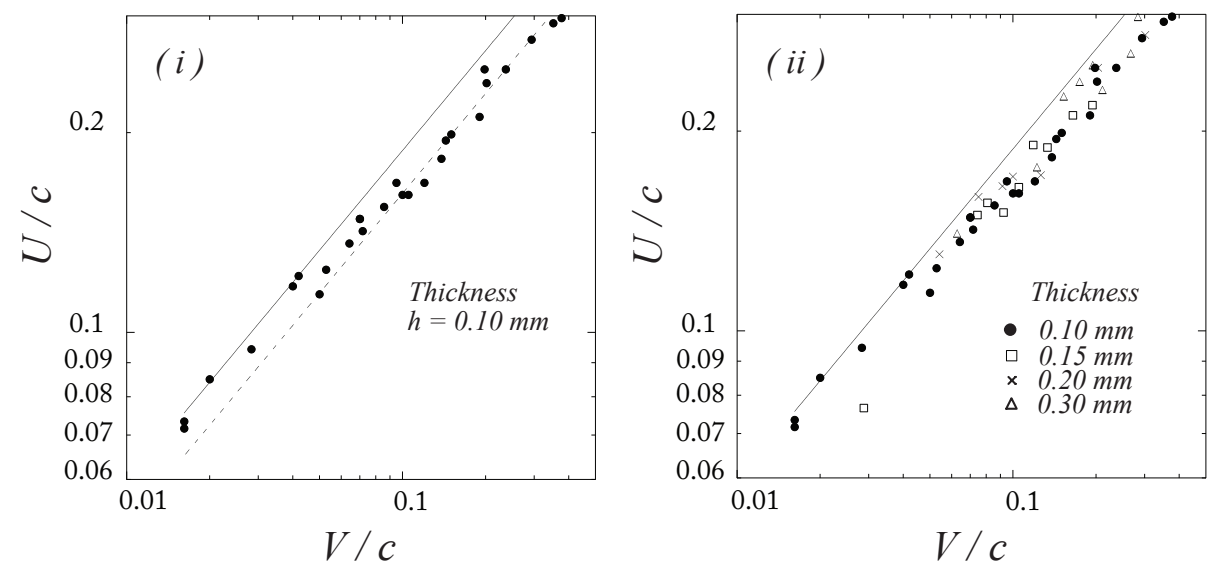

Figure 8. Transverse front velocity vs impact velocity. Velocity $U$ in the lagrangian frame were deduced from the measurements of the transverse front velocity in the laboratory frame $\bar{U}$. (i) Measurements for a latex sheet of thickness $h=0.10 \mathrm{~mm}$. The solid line stands for the theoretical prediction with no adjustable parameter, the dashed line stands for the fit by the theoretical curve with an adjustable prefactor, we observe a $30 \%$ error on the prefactor. (ii) Measurements for latex sheet of different thicknesses..

(c) Stress field resulting from an impact on a 2D elastic membrane

We use the approximation of equation (4.9) for the radial displacement to compute the stress field in the stretched area. With $\alpha=(1 / 4)(V / c)^{2}$, we have

$$
\begin{aligned}
\sigma_{r}(r, t) & =\frac{E}{1-\nu^{2}}\left(\frac{V}{2 c}\right)^{2}\left[1+\nu+(1-\nu)\left(\frac{r_{i}+c t}{r}\right)^{2}\right] \\
\sigma_{\theta}(r, t) & =\frac{E}{1-\nu^{2}}\left(\frac{V}{2 c}\right)^{2}\left[1+\nu-(1-\nu)\left(\frac{r_{i}+c t}{r}\right)^{2}\right]
\end{aligned}
$$

These expressions are valid in the domain between $r_{c}(t)=r_{i}+U t$ and $r_{t}(t)=$ $r_{i}+c t$. The circumferential stress becomes negative at $r=r_{z}(t)=[(1-\nu) /(1+$ $\nu)]^{1 / 2}\left(r_{i}+c t\right)$. Then the area of the plate delimited by the transverse wave front $r=r_{c}(t)$ and $r=r_{z}(t)$ is compressed along the circumferential direction (figure 9). This compression results from a geometrical constrain: consider two points initially at radius $r$ and at angles $\theta_{1}$ and $\theta_{2}$. Initially the arc length between these two points is $r\left(\theta_{2}-\theta_{1}\right)$. After deformation this distance is reduced to $[r+\zeta(r, t)]\left(\theta_{2}-\theta_{1}\right)$ where $\zeta(r, t)$ is negative.

\section{Dynamic wrinkling}

In thin sheets, compressive in plane stresses lead to the formation of wrinkles. In this section, we study the appearance of wrinkles and we compute their size. As usual for buckling instabilities, this size results from a balance between destabilization induced by the circumferential compression, and restoring forces due to the sheet 

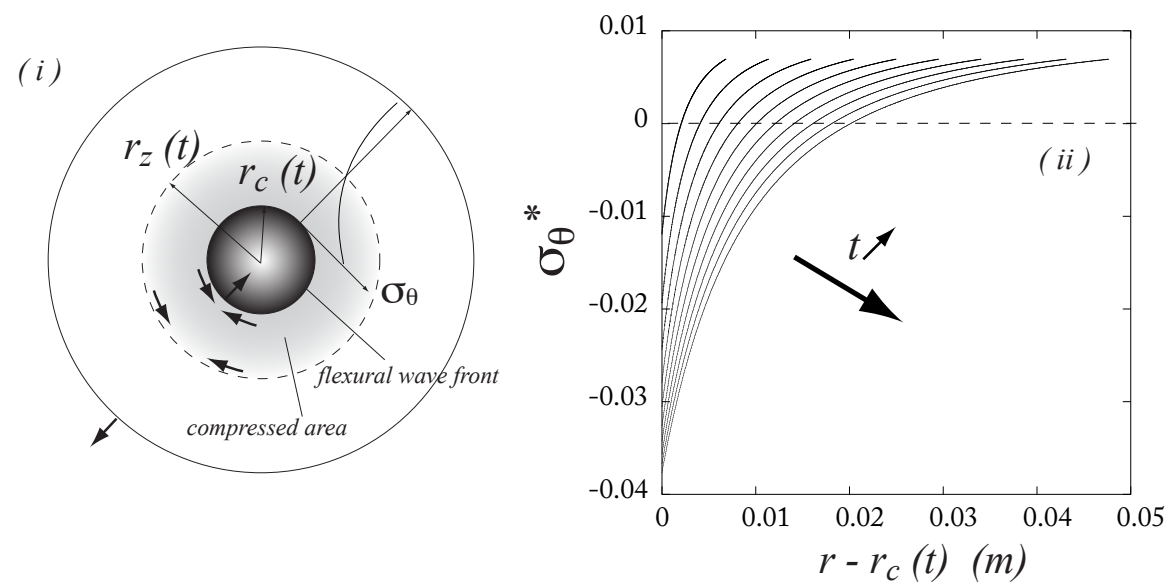

Figure 9. (i) Schematic showing the stress field in the stretched area consecutive to an impact on an elastic membrane. The central dark area is the cone. The shaded area represents the region $r_{c}(t)<r<r_{z}(t)$ where circumferential stress is positive. (ii) Variation of the normalized circumferential stress $\sigma_{\theta}^{*}=\sigma_{\theta} /\left(E /\left(1-\nu^{2}\right)\right)$ with the distance to the transverse wave front. The dashed line shows the position where the stress changes sign. The stresses are calculated for a membrane impacted at velocity $V=5 \mathrm{~m} \mathrm{~s}^{-1}$ by a $4.5 \mathrm{~mm}$ large impactor and for times ranging from $0.3 \mathrm{~ms}$ to $2.1 \mathrm{~ms}$ after impact.

bending rigidity. To model the behavior of the compressed area, we investigate the analog problem of the stability of an annular plate, of inner radius $r_{c}(t)$ and outer radius $r_{z}(t)$, to which is applied the in-plane stress field (both radial and circumferential) found in section $4 \mathrm{c}$. The problem is similar to the static problem studied by Géminard et al. (2004). Coman \& Haughton (2006) also proposed a theoretical analysis of the problem. These authors studied the stability of the plan solution and computed the shape of the unstable mode as a function of $r$ and $\theta$. We adopt a global method imposing the shape of the pattern, providing the instability threshold and azimuthal wavelength. Later on we also discuss a simplified model, approximating the annulus with a beam, resembling the method used by Senior (1956) in the context of wrinkling in metal punching but with added inertia.

\section{(a) Instability of the stretched membrane}

The Rayleigh method (Rayleigh 1894) is an application of the Least Action Principle to vibrating systems whose dynamics is on purpose restricted to one degree of freedom. The method consists in making the inventory of the elastic energies involved, namely the bending energy $U_{b}$, the circumferential compressive energy $U_{c}$, the radial tensile energy $U_{t}$, construct a potential $U=U_{b}+U_{c}+U_{t}$ and then write

$$
\delta \int \mathrm{d} t(T-U)=0
$$

where $T$ is the sheet kinetic energy, and $\delta$ stands for a variation in the displacements $\xi_{n}$ that make the integral minimum. The optimum provides the dispersion relation of the problem for given in-plane stresses $\sigma_{r}$ and $\sigma_{\theta}$. We consider a single radial 
bent, imposing $\xi=0$ at the inner radius $r=r_{c}(t)$ and outer radius $r=r_{z}(t)$ and account for $n$ folds in the circumferential direction as

$$
\xi(r, \theta, t)=\frac{\left(r-r_{c}\right)\left(r-r_{z}\right)}{\left(r_{z}-r_{c}\right)^{2}} \xi_{n} \sin (n \theta) \mathrm{e}^{\gamma_{n} t}
$$

where $\xi_{n}$ and $\gamma_{n}$ are respectively the amplitude and the growth rate. This form assumes that the out of plane displacement for $r>r_{z}$ is zero.

- The expression for the bending energy is

$$
\begin{aligned}
U_{b}=\frac{D}{2} \int_{0}^{2 \pi} \int_{r_{c}}^{r_{z}}\left\{\left(\frac{\partial^{2} \xi}{\partial r^{2}}+\right.\right. & \left.\frac{1}{r} \frac{\partial \xi}{\partial r}+\frac{1}{r^{2}} \frac{\partial^{2} \xi}{\partial \theta^{2}}\right)^{2} \\
-2(1-\nu) & \left(\frac{\partial^{2} \xi}{\partial r^{2}}\right)\left(\frac{1}{r} \frac{\partial \xi}{\partial r}+\frac{1}{r^{2}} \frac{\partial^{2} \xi}{\partial \theta^{2}}\right) \\
& \left.+2(1-\nu)\left(\frac{\partial}{\partial r}\left(\frac{1}{r} \frac{\partial \xi}{\partial \theta}\right)\right)^{2}\right\} r d r d \theta
\end{aligned}
$$

where $D=E h^{3} /\left[12\left(1-\nu^{2}\right)\right]$ is the bending rigidity (Timoshenko \& WoinowskyKrieger 1959). With $\xi$ given by equation (5.2), the bending energy writes

$$
\begin{aligned}
U_{b}=\frac{\pi D \xi_{n}^{2} \mathrm{e}^{2 \gamma_{n} t}}{2\left(r_{z}-r_{c}\right)^{4}}\{ & {\left[-3\left(r_{z}^{2}-r_{c}^{2}\right)+\left(r_{c}^{2}+4 r_{c} r_{z}+r_{z}^{2}\right) \ln \frac{r_{z}}{r_{c}}\right] n^{4} } \\
+ & 2\left[4\left(r_{z}^{2}-r_{c}^{2}\right)-\left(r_{c}^{2}+6 r_{c} r_{z}+r_{z}^{2}\right) \ln \frac{r_{z}}{r_{c}}\right] n^{2} \\
+ & {\left.\left[\left(r_{c}+r_{z}\right)^{2} \ln \frac{r_{z}}{r_{c}}\right]\right\} }
\end{aligned}
$$

- In-plane stresses also contribute to the elastic energy of the system. The compressive circumferential stress $\sigma_{\theta}$ yields a compressive energy $U_{c}$ while the radial stress $\sigma_{r}$ yields a tension energy $U_{t}$. They are given by

$$
\begin{aligned}
U_{c} & =\frac{h}{2} \int_{0}^{2 \pi} \int_{r_{c}}^{r_{z}}\left\{\sigma_{\theta}\left(\frac{1}{r} \frac{\partial \xi}{\partial \theta}\right)^{2}\right\} r d r d \theta \\
U_{t} & =\frac{h}{2} \int_{0}^{2 \pi} \int_{r_{c}}^{r_{z}}\left\{\sigma_{r}\left(\frac{\partial \xi}{\partial r}\right)^{2}\right\} r d r d \theta
\end{aligned}
$$

The compressive and tensile energies thus write

$$
\begin{aligned}
U_{c} & =\frac{\pi D \xi_{n}^{2} \mathrm{e}^{2 \gamma_{n} t} n^{2}}{2 h^{2}\left(r_{c}-r_{z}\right)^{4}}\left(\frac{V}{2 c}\right)^{2} I_{c} \\
U_{t} & =\frac{\pi D \xi_{n}^{2} \mathrm{e}^{2 \gamma_{n} t}}{h^{2}\left(r_{c}-r_{z}\right)^{4}}\left(\frac{V}{2 c}\right)^{2} I_{t}
\end{aligned}
$$

with

$$
\begin{gathered}
I_{c}=\frac{1}{2}\left\{(1-\nu)\left(r_{i}+c t\right)^{2}\left[36\left(r_{z}^{2}-r_{c}^{2}\right)-12\left(r_{z}^{2}+4 r_{c} r_{z}+r_{c}^{2}\right) \ln \frac{r_{z}}{r_{c}}\right]\right. \\
\left.+(1+\nu)\left[12 r_{c}^{2} r_{z}^{2} \ln \frac{r_{z}}{r_{c}}+\left(r_{z}^{2}-r_{c}^{2}\right)\left(r_{c}^{2}-8 r_{c} r_{z}+r_{z}^{2}\right)\right]\right\}
\end{gathered}
$$


and

$$
I_{t}=(1-\nu)\left(r_{i}+c t\right)^{2}\left[6\left(r_{c}+r_{z}\right)^{2} \ln \frac{r_{z}}{r_{c}}-12\left(r_{z}^{2}-r_{c}^{2}\right)\right]+(1+\nu)\left(r_{z}^{2}-r_{c}^{2}\right)^{2}
$$

- Finally the kinetic energy is given by

$$
T=\frac{\rho h}{2} \int_{0}^{2 \pi} \int_{r_{c}}^{r_{z}}\left\{\left(\frac{\partial \xi}{\partial t}\right)^{2}\right\} r d r d \theta
$$

Using $\rho=12 D /\left(c^{2} h^{3}\right)$, we have

$$
T=\frac{\pi D \xi_{n}^{2} \gamma_{n}^{2} e^{2 \gamma_{n} t}}{10 c^{2} h^{2}}\left(r_{z}^{2}-r_{c}^{2}\right)
$$

Then, using (5.1), we find the dispersion equation for the circumferential wave number $n$, which we arrange to obtain that corresponding to the dimensional circumferential wave number calculated at the inner radius $k_{n}=n / r_{c}$

$$
\begin{gathered}
\frac{\left(r_{z}-r_{c}\right)^{4}\left(r_{z}^{2}-r_{c}^{2}\right)}{10 c^{2} h^{2}} \gamma_{n}^{2}+\frac{1}{2} k_{n}^{4} r_{c}^{4}\left[-3\left(r_{z}^{2}-r_{c}^{2}\right)+\left(r_{c}^{2}+4 r_{c} r_{z}+r_{z}^{2}\right) \ln \frac{r_{z}}{r_{c}}\right] \\
+k_{n}^{2} r_{c}^{2}\left[4\left(r_{z}^{2}-r_{c}^{2}\right)-\left(r_{c}^{2}+6 r_{c} r_{z}+r_{z}^{2}\right) \ln \frac{r_{z}}{r_{c}}+\frac{1}{2}\left(\frac{V}{2 c}\right)^{2} \frac{I_{c}}{h^{2}}\right] \\
+\frac{1}{2}\left(r_{c}^{2}+r_{z}^{2}\right) \ln \frac{r_{z}}{r_{c}}+\left(\frac{V}{2 c}\right)^{2} \frac{I_{t}}{h^{2}}=0
\end{gathered}
$$

The second term (proportional to $k_{n}^{4}$ ) is always positive and thus at short wavelength, $\gamma_{n}^{2}$ is negative and the solution is stable. The source of the instability is the term proportional to $I_{c}$. Substituting time dependence for the radius $r_{z}$ and $r_{c}$, we obtain a dispersion equation dependent on time (figure 10). At a critical time $t_{\text {inst }}$, the sign of $\gamma_{n}^{2}$ changes and the axisymmetric solution becomes unstable. A wavelength is selected by the instability.

The critical time at which the axisymmetric solution becomes unstable can be computed by looking for a double root of the equation in $k_{n}$ obtained by looking at solutions $\gamma_{n}=0$. A wavelength is associated with this critical time. Comparaison between the wavelength computed from equation (5.13) and the experimental wavelength shows a good agreement (figure 11). However for very thin sheets and high impacting speeds (figure 11a), the instability develops at early times and the approximation $r \gg r_{i}$ used to obtain equation (4.19) is no longer valid.

We also measured the time at which we start to observe wrinkling (figure 12). This time is not stricto sensu equal to $t_{\text {inst }}$ because the wrinkles need time to grow whereas at the instability threshold (i.e. at time $t_{\text {inst }}$ ), the growth rate is zero. Above $t_{\text {inst }}$, the maximal growth rate $\gamma$ increases itself in time (figure 10b) and saturates over a time period of about $10 \mathrm{~h} / \mathrm{V}$. Figure 12 shows that the typical wrinkling time is indeed $t_{\text {inst }}+10 \mathrm{~h} / \mathrm{V}$.

\section{(b) Approximation of the instability wavelength}

An explicit form for the critical time $t_{\text {inst }}$ and the instability wavelength $\lambda$ cannot be easily computed from equation (5.13). In the limit $r_{i} \rightarrow 0$ and assuming 

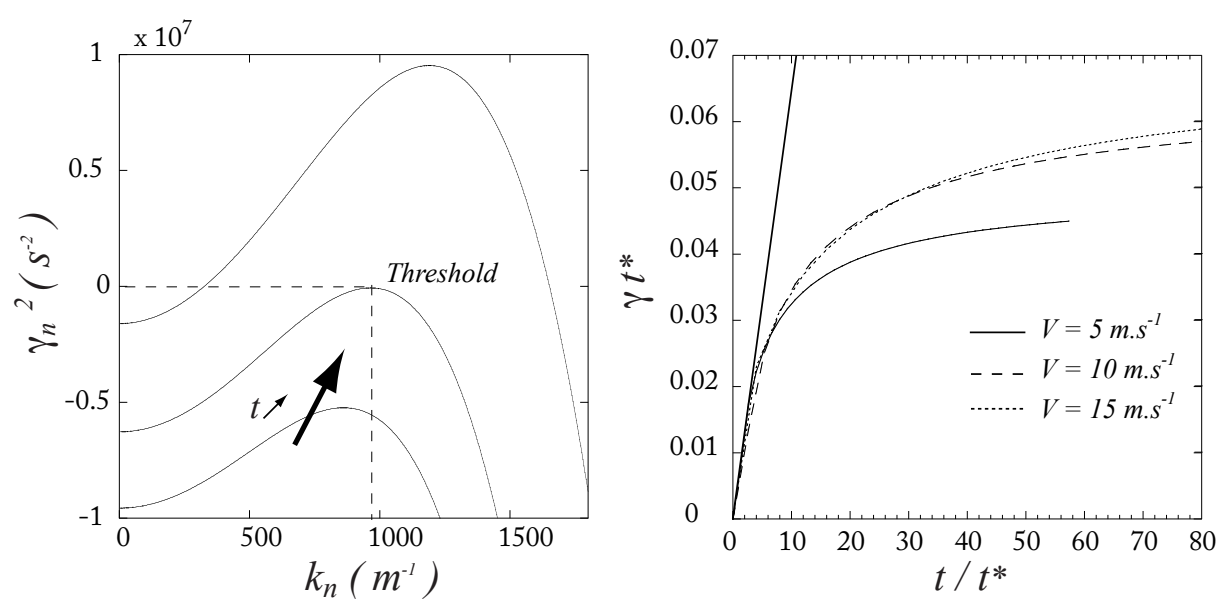

Figure 10. (i) Variation of the instability growth rate with the wavenumber. The dispersion relation was calculated numerically for a projectile of radius $r_{i}=2.25 \mathrm{~mm}$ impacting a plate of thickness $h=0.15 \mathrm{~mm}$ at impacting velocity $V=10 \mathrm{~m} \mathrm{~s}^{-1}$. The dispersion relation is calculated for several times after impact: $300 \mu \mathrm{s}, 365 \mu \mathrm{s}$ and $700 \mu \mathrm{s}$. We observe a finite wavenumber at the instability threshold. (ii) Time evolution of the optimal growth rate $\gamma$ as a function of time. Times are normalized by $t^{*}=h / v ; h=0.15 \mathrm{~mm}$.

that $V / c$ is small, the instability occurs at large times. The compressed area shown on figure 9 has a large radius and its curvature can be neglected, hence we propose the following toy model to understand the instability mechanism: consider an elastic beam of length $\pi\left[r_{z}(t)+r_{c}(t)\right]$ and width $b=r_{z}(t)-r_{c}(t)$ with a compression

$$
\sigma_{c}=\sigma_{\theta}\left(r_{c}\right)=\frac{E}{1-\nu^{2}}\left(\frac{V}{2 c}\right)^{2}\left[(1+\nu)-2 \sqrt{1-\nu} \frac{c}{V}\right] \approx-\frac{E}{1-\nu^{2}} \sqrt{1-\nu} \frac{V}{2 c}
$$

The dispersion equation (Lindberg 1965 and Vermorel \& al. 2007) is

$$
\omega^{2}=\frac{E I}{\rho b h} k^{2}\left\{k^{2}+\sigma_{c} \frac{b h}{E I}\right\}
$$

where $I=b h^{3} / 12$ is the flexural inertia momentum. The straight configuration of the beam is unstable and a dynamic buckling instability develops. This instability selects a most amplified wavelength

$$
\lambda_{\max }=\pi h \sqrt{\frac{2}{3}} \sqrt{\frac{E}{\left|\sigma_{c}\right|}} \approx \frac{2 \sqrt{3}}{3} \pi(1+\nu)^{1 / 2}(1-\nu)^{1 / 4} h\left(\frac{V}{c}\right)^{-1 / 2}
$$

The scaling above $\lambda \propto h(V / c)^{-1 / 2}$ is precisely the one observed experimentally for moderately thin sheets (figure $11(i)$ ). The instability time of growth is

$$
\tau_{\text {growth }}=\frac{\sqrt{3}}{3} h \frac{1}{c} \frac{E}{\left|\sigma_{c}\right|} \approx 2 \frac{\sqrt{3}}{3}\left(1-\nu^{2}\right) \frac{h}{V}
$$

whose scaling again recovers that of $t_{\text {inst }}$. 

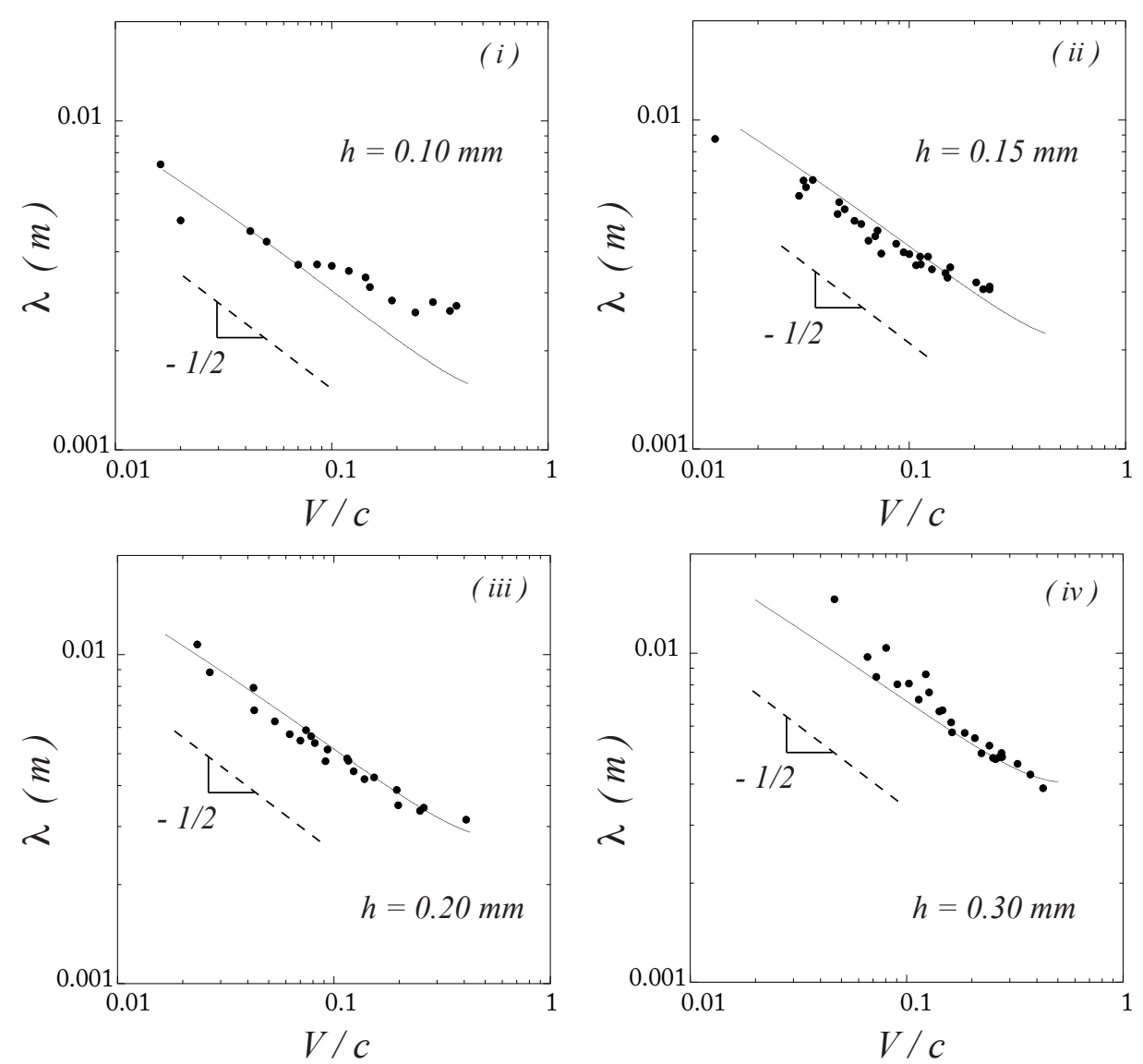

Figure 11. Variation of the buckled wavelength with the impacting velocity. We conducted experiments with a projectile of radius $r_{i}=2.25 \mathrm{~mm}$ and latex sheets of different thicknesses. The solid lines stand for the theoretical predictions with no adjustable parameter.

\section{Conclusion and discussion}

\section{(a) Summary}

We have shown that a transversely impacted thin sheet experiences a buckling instability leading to the growth of radial wrinkles. The instability, which results from the "geometrical confinement" of material points in the sheet focusing towards the impact point, selects a wavelength correctly predicted by a quasistatic analysis of a time-varying substrate. Interestingly, this instability is not observed in clamped circular sheets with a central transverse force. On the other hand this instability occurs in membranes which are geometrically constrained at one or two different radii like in Géminard et al. (2004), Huang et al. (2007) or Chopin et al. (2008). The necessary confinement occurs in the present case because the tensile wave front propagates faster than the transverse wave front. The main difference with the static case concerns the wrinkle pattern: in the static case a number of wrinkles 

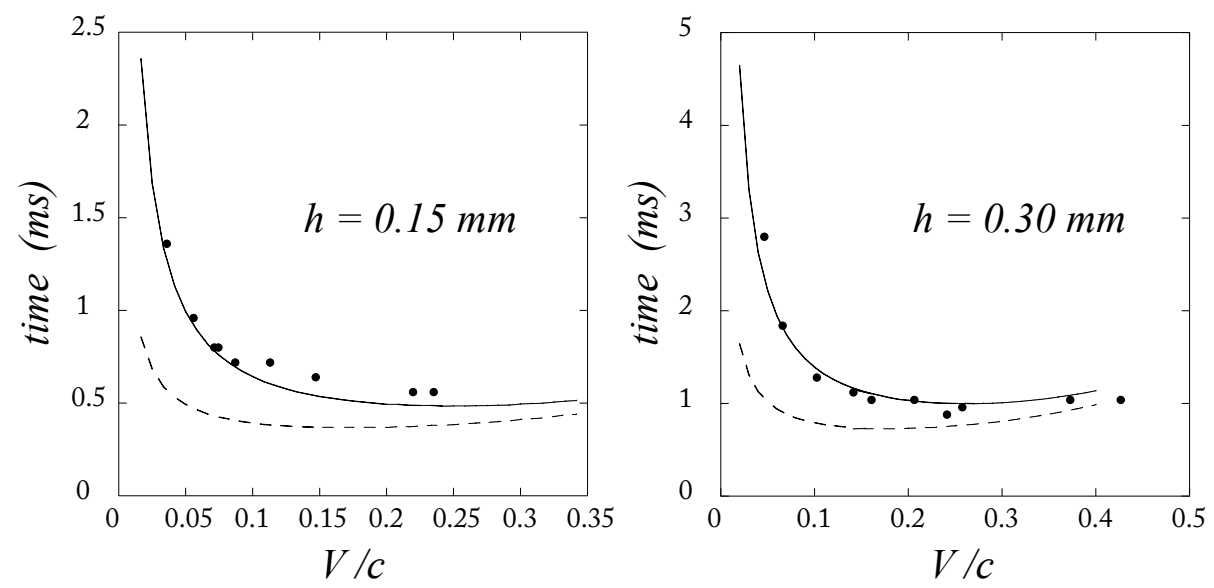

Figure 12. Development time of the wrinkling instability observed experimentally as a function of the impacting velocity. Dashed line: contribution of $t_{i n s t}$ only, Solid line, $t_{\text {inst }}+10 h / V$ (see text).

is selected and the wavelength changes with the distance from the center while here the wavelength is conserved.

We also note the similarity between the patterns resulting from this wrinkling instability and the cracks which are observed on impacted plates of brittle materials, whose analysis is left for future work.

Modification of geometry, boundary conditions impact parameters or material properties may only modify the critical radius (and critical time) at which the instability develops. Thus our model does not predict a threshold for the buckling instability. In the following we discuss different limitations of our model. In particular we discuss the effects of the inertia of the impactor, of the size of the latex sheet and of the sheet thickness.

\section{(b) Deceleration of the impacting body}

We assumed that the velocity of the impactor $V$ does not change during impact. If the mass of the impactor is finite however, it decelerates. Using the results of section $4 \mathrm{~b}$, the rate of decrease of the impactor's velocity can be estimated from momentum conservation of the system composed of the impactor and the mass of the membrane displaced vertically.

Consider a rigid impactor of mass $M_{p}$, impacting an elastic sheet at initial impacting velocity $V$. The part of the sheet whose motion is vertical is the area delimited by the transverse wave front resulting from impact. The vertical velocity of the membrane at the impact point is the same as the impactor current velocity $v(t)$. As an approximation, we consider that the transverse wave front velocity only depends on the initial impacting velocity $V$ as if the impactor had an infinite mass and we use the result from (4.19). Then, the mass of the membrane moving vertically is

$$
M_{m}=\rho h \pi(U t)^{2}=\pi \rho h\left(\frac{1-\nu}{4}\right)^{1 / 2}\left(\frac{V}{c}\right) c^{2} t^{2}
$$



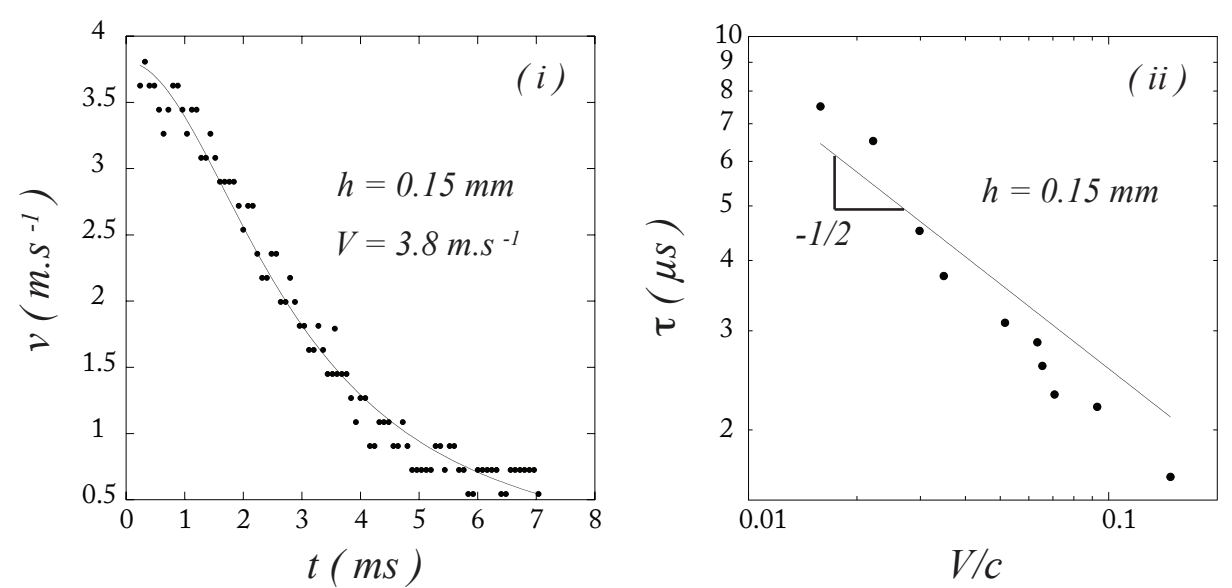

Figure 13. (i) Variation of the velocity of the impactor (a steel ball of mass $m_{p}=0.33 \times 10^{-3} \mathrm{~kg}$ ) with time. The solid line stands for the fitting curve with one adjustable parameter evaluating the characteristic time $\tau$. The data fit provides $\tau=2.92 \mathrm{~ms}$ while the theoretical value is $2.9 \mathrm{~ms}$. (ii) Variation of the characteristic time $\tau$ with the impacting velocity. The solid line stands for the fit by the theoretical power law $(V / c)^{-1 / 2}$. The error on the prefactor does not exceed $10 \%$.

Conservation of momentum in the vertical direction writes as

$$
\frac{d}{d t}\left\{\left(M_{p}+M_{m}\right) v\right\}=\left(M_{p}+M_{m}\right) \frac{d v}{d t}+v \frac{d M_{m}}{d t}=0
$$

Hence the differential equation satisfied by $v(t)$

$$
\frac{d v}{d t}=-\left(\frac{2 t}{t^{2}+\tau^{2}}\right) v, \quad \text { with } \quad \tau^{2}=\frac{M_{p}}{\pi \rho h\left(\frac{1-\nu}{4}\right)^{1 / 2}\left(\frac{V}{c}\right) c^{2}}
$$

which integrates into

$$
v(t)=\frac{V}{1+(t / \tau)^{2}}
$$

Measurements of the impactor velocity $v(t)$ and of the characteristic deceleration time $\tau$ show in Figure 13 a good agreement with (6.4). The typical observation time in the present experiments does not exceed $2 \mathrm{~ms}$ after time of impact. The mass of the impactor associated to $\tau=2 \mathrm{~ms}$ for a membrane thickness $h=0.30 \mathrm{~mm}$ and for an impacting velocity $V=30 \mathrm{~m} \mathrm{~s}^{-1}$ is $M_{p}=2.4 \times 10^{-3} \mathrm{~kg}$. The experiments presented in this work were all conducted with $M_{p}=3.3 \times 10^{-3} \mathrm{~kg}$ or larger, masses for which deceleration does not alter our conclusions.

\section{(c) Finite size effects}

According to the dispersion equation (5.13), the instability develops for any impacting speed. However finite size limitations can modify the dynamics. The tension front reaches the extremity of the sheet in $r=r_{0}$ at time $t_{0}=r_{0} / c$. This front rebounds and if it reaches the position $r=r_{z}$ before the development of the 
instability, the stress field changes and we expect a modified dynamics. Thus the critical size for the membrane is given by the criterion

$$
\frac{r_{i}-r_{0}}{c}+\frac{r_{0}-r_{z}\left(t_{i n s t}\right)}{c}<t_{\text {inst }}
$$

The measurements in the present experiments (wave speed, radial displacements, wavelengths...) were performed before perturbation by the rebounding front. We also note that the shape of the rebounding front depends on the boundary conditions (free, clamped ...). However as long as there is no interaction with the rebounding wavefronts, the boundary conditions do not influence the dynamics and the development of the instability. This is a difference with the static situation where the boundary conditions are fundamental in predicting the onset of wrinkling.

\section{(d) Pure bending waves}

Finally our model for transverse wave propagation disregards the dynamics of pure bending waves, decoupled from tension in the sheet. We briefly discuss the validity of this model in the one dimensional case. If flexural stiffness is taken into account equation (4.1) now reads

$$
E A r^{2} \frac{\partial^{4} \xi}{\partial x^{4}}+\frac{\partial}{\partial x}\left(T \frac{\partial \xi}{\partial c}\right)+\rho A \frac{\partial^{2} \xi}{\partial t^{2}}=0
$$

where $r$ is the radius of gyration of the beam $\left(r^{2}=h^{2} / 12\right.$ for a rectangular cross section of thickness $h$ ) and $T=E A \epsilon$. We note that this equation is valid at times larger than $h / c$. When the beam is impacted, transverse waves propagate as bending waves and tension increases gradually as a consequence of transverse motion. Consequently, any localized disturbance, say $\xi(r, t=0)=f(r)$ will propagate according to $(6.6)$ as a wave packet $\xi(r, t)=F(r / \sqrt{\kappa t})$ where $\kappa=c r$ and $F(\cdot)$ is an eigenfunction of (6.6) (see e.g. Graff 1975). The wave packet thus expands over a typical width increasing like $\sqrt{\kappa t}$. Now, the tension front sustained by the continuing traction of the impactor has propagated radially over a distance $c t$ at time $t$. Thus assuming that the tensile strain is uniform over the stretched domain we estimate the strain in the beam as follows: the segment of initial length $c t$ now has a length of $c t-\sqrt{\kappa t}+\left[\kappa t+(V t)^{2}\right]^{1 / 2}$ and thus the strain is

$$
\epsilon=\frac{c t-\sqrt{\kappa t}+\left[\kappa t+(V t)^{2}\right]^{1 / 2}-c t}{c t} \approx \frac{1}{2}\left(\frac{V}{c}\right)^{2} \frac{c t}{\sqrt{\kappa t}}
$$

Using this estimate, we compare the two first terms in equation (6.6): the bending term writes $E A r^{2}(V t) /(\kappa t)^{2}$ and the tension term writes $E A(V / c)(c t / \sqrt{\kappa t}) V t / \kappa t$. Thus the bending term dominates until a critical time

$$
t_{b}=\frac{r}{V}\left(\frac{c}{V}\right)^{1 / 3} \propto \frac{h}{V}\left(\frac{c}{V}\right)^{1 / 3}
$$

above which the restoring force due to tension overcomes the intrinsic bending rigidity. This time is greater than $h / V$ and thus the impactor must have travelled a distance greater than $h$ for the tension to be large enough. In $2 \mathrm{D}$ we expect the same limitation but in our experiments $h / V$ (typically $10^{-5} \mathrm{~s}$ ) is very short compared to observation times; our model neglecting bending rigidity is valid. 


\section{References}

Backmann, M. A. \& Goldsmith, W. 1978 The mechanics of penetration of projectiles into target. Int. J. Eng. Sci. 16, 1-99.

Begley, M. R. and Mackin, T. J. 2004 Spherical indentation of freestanding circular thin films in the membrane regime. J. Mech. Phys. Solids 52, 2005 - 2023.

Bouasse, H. \& Carrière, Z. 1903 Sur les courbes de traction du caoutchouc vulcanisé. Annales de la faculté des sciences de Toulouse, 2e série 5, 257-283.

Cerda, E., Mahadevan, L. \& Pasini, J.M. 2004 The elements of draping. Proc. Natl. Acad. Sci. USA 101, 1806-1810.

Chopin, J., Vella, D. \& Boudaoud, A. 2008 The liquid blister test. Proc. R. Soc. A., doi:10.1098/rspa.2008.0095.

Coman, C. D. \& Haughton, D. M. 2006 Localized wrinkling instabilities in radially stretched annular thin films. Acta Mech., 185, 179-200.

Farrar, C. 1984 Impact response of a circular membrane. Exp. Mech. 24, 144-149.

Géminard, J.-C., Bernal, R. \& Melo, F. 2004 Wrinkle formations in axi-symmetrically stretched membranes. Eur. Phys. J. E 15, 117-126.

Graff, K. G. 1975 Wave motion in elastic solids. New York, Dover Publications.

Huang,J., Juszkiewicz, M., de Jeu, w. H., Cerda,, E., Emrick, T. Menon, N. \& Russell, T. P. 2007 Capillary wrinkling of floating thin polymer films, Science, 317, 650-653.

Lindberg, H. E. 1965 Impact buckling of a bar. J. Appl. Mech. 32, 315-322

Love, A. E. H. 1944. A treatise on the mathematical theory of elasticity. 4th edn. New York: Dover Publications.

Mullins, L. 1947 Effect of stretching on the properties of rubber. J. Rubber Research 16, 275-289.

Phoenix, S. L. \& Porwal, P. K. 2003 A new membrane model for the ballistic impact response and V50 performance of multi-ply fibrous systems. Int. J. Solids Struct. 40, 6723-6765.

Rayleigh, J. W. S. 1894 The theory of Sound. New York, Dover Publications.

Senior, B. W. 1956 Flange wrinkling in deep-drawing operations. J. Mech. Phys. Solids 4, 235-246.

Timoshenko, S. \& Woinowsky-Krieger, S. 1959 Theory of plates and shells. New York, McGraw-Hill.

Vermorel, R., Vandenberghe, N. \& Villermaux, E. 2007 Rubber Band Recoil. Proc. R. Soc. A 463, 641-658. 